In Tab. 1 ist das Ergebnis der einzelnen Reinigungsschritte am Beispiel von Hundeleber eingetragen.

Wir verwendeten Hundeleber als Ausgangsmaterial, weil die GPT-Aktivität hier etwa 100 -fach höher ist als in Rinder- und Schweineleber. In Kalbsleber ist die GPT-Aktivität ebenfalls sehr gering.

II. Wirkung des hochgereinigten GPT-Präparates a uf die anaerobe Glykolyse von EMAC-Zellen

Es wurden alle GPT-Präparate aus den einzelnen Reinigungsschritten auf ihre Glykolyse-hemmende Wirkung bei EMAC-Zellen geprüft. In Tab. 2 sind die Ergebnisse zusammengefaßt. Wie man sieht, bleibt, unabhängig vom Reinheitsgrad, mit fortschreitender Reinigung der GPT die hemmende Wir- kung auf die Glykolyse erhalten. Insbesondere ist das hochgereinigte GPT-Präparat gleich wirksam wie Leberextrakt, wenn der Test mit der gleichen Konzentration an GPT-Einheiten durchgeführt wird. Damit sind die Schlußfolgerungen der früheren Arbeit ${ }^{1}$ mit Leberextrakt bestätigt, daß die hemmende Wirkung des Leberextraktes auf die anaerobe Glykolyse von EMAC-Zellen auf die Wirkung der im Leberextrakt enthaltenen Glutamat-Pyruvat-Transaminase zurückzuführen ist.

Frau Ute MarquaRdT danke ich für ihre wertvolle Mitarbeit bei der technischen Durchführung der Versuche.

15 G. Beisenherz, H. J. Boltze, Th. Bücher, R. Czok, K. H. Garbade, E. Meyer-Arendt u. G. Pfleiderer, Z. Naturforschg. 8 b, 555 [1953].

\title{
Zur Lokalisation des Myosins in den Muskelfasern aus dem Haut- muskelschlauch des Regenwurms *
}

\author{
H.-G. Heumann und E. Zebe \\ Zoologisches Institut der Universität Heidelberg, Physiologischer Lehrstuhl \\ (Direktor: Prof. Dr. F. Duspiva)
}

(Z. Naturforschg. 21 b, 62-65 [1966] ; eingegangen am 18. August 1965)

\begin{abstract}
Die Lokalisation ATP-spaltender Reaktionen in der Feinstruktur von Fasern aus dem Hautmuskelschlauch von Lumbricus terrestris wurde an mit Formol vorbehandelten und z. T. mit Glycerin extrahierten Präparaten studiert. Dabei konnte demonstriert werden, daß die Fällung des enzymatisch freigesetzten anorganischen Phosphats durch $\mathrm{Pb}^{2 \oplus}$ ausschließlich in unmittelbarer Nähe der dicken Filamente stattfindet. Die Niederschlagspartikel liegen vorzugsweise in Reihen zwischen den Einzelfilamenten und zeigen eine auffällige Massierung in deren zentralen Bereichen. Die Endabschnitte enthalten dagegen viel weniger Präzipitat. Diese Niederschlagsverteilung ist unabhängig vom Kontraktionszustand.

Die ATP-Spaltung durch die dicken Filamente wird als Anzeichen für die Lokalisation von Myosin in diesen gedeutet und unter vergleichenden Gesichtspunkten diskutiert.
\end{abstract}

In Übereinstimmung mit dem wohlbekannten Aufbau der quergestreiften Muskelfasern aus Myosinund Aktinfilamenten ${ }^{1}$ ist auch für einige andere Muskeltypen die Existenz von zwei charakteristisch verschiedenen Arten von Myofilamenten nachgewiesen worden ${ }^{2-8}$. Bei manchen glatten Muskeln war allerdings die Unterscheidung von zwei Filamenten

* Mit dankenswerter Unterstützung durch die D e u t s c h e Forschungs gemeins chaft.

1 H. E. Huxley, J. biophysic. biochem. Cytol. 3, 631 [1957].

2 J. Hanson u. J. Lowy, Proc. Royal Soc. [London], Ser. B 154, 173 [1961].

3 S. Kawaguti, Proc. 5th Int. Congr. Electron Microscopy 2, M-11 [1962].

${ }^{4}$ B. A. Afzelius, Proc. 5th Int. Congr. Electron Microscopy 2, M-1 [1962].

5 J. K. Chог, Proc. 5th Int. Congr. Electron Microscopy 2, M-9 [1962].

6 N. Iкемото, Biol. J. Okayama Univ. 9, 81 [1963].

7 E. K. Macrae, J. Cell Biol. 18, 651 [1963]. auf Grund rein morphologischer Merkmale bisher nicht möglich ${ }^{9}$. Dennoch liegt die Annahme nahe, daß die kontraktilen Proteine bei allen nichtquergestreiften Muskeln ebenso getrennt voneinander angeordnet und jeweils in einem bestimmten Filamenttyp lokalisiert sind, wie dies für die quergestreiften Muskeln erwiesen ist ${ }^{10-15}$. Dafür spricht die Wahr-

8 J. Rosenbluth, J. Cell Biol. 25/3, 495 [1965].

9 C. F. Shoenberg, Proc. 5th Int. Congr. Electron Mic:oscopy 2, M-8 [1962].

10 H. E. Huxley u. J. Hanson, Biochim. biophysica Acta [Amsterdam] 23, 229 [1957].

11 J. Hanson u. H. E. Huxley, Biochim. biophysica Acta [Amsterdam] 23, 250 [1957].

12 H. Finck, H. Holtzer u. J. M. Marshall, J. biophysic. biochem. Cytol. 2, Suppl. 175 [1956].

13 B. Tunik u. H. Holtzer, J. biophysic. biochem. Cytol. 11, 67 [1961].

14 E. Zebe u. H. Falk, Z. Naturforschg. 18 b, 501 [1963].

15 E. Z ZBe u. H. FALK, Histochemie 4, 161 [1964]. 
scheinlichkeit, daß der Kontraktilität aller Muskeln ein einheitliches Prinzip zugrunde liegt. Das stärkste Argument für einen solchen Sachverhalt liefern Beobachtungen, wonach bei einigen nichtquergestreiften Muskeln bei der Kontraktion die dünnen Filamente zwischen die dicken gezogen werden, die Verkürzung hier also anscheinend wie bei den quergestreiften Muskeln durch einen Gleitmechanismus erfolgt ${ }^{2,6,16}$. Hanson und Lowy konnten darüber hinaus an Muskeln der Mollusken auch noch die Existenz von Querbrücken zwischen den dicken und dünnen Filamenten demonstrieren ${ }^{2}$, wie sie von Huxley an den Skelettmuskeln der Säuger entdeckt wurden ${ }^{1}$.

Wir haben nun an Fasern aus dem Hautmuskelschlauch des Regenwurms Lumbricus terrestris Versuche zur Lokalisation des Myosins durchgeführt und dabei Ergebnisse erhalten, die in diesem $\mathrm{Zu}$ sammenhang von besonderem Interesse sind. Sie bestätigen die prinzipielle Übereinstimmung dieser „helikalen“ oder „schräggestreiften“ Muskeln mit der Organisation des kontraktilen Apparats bei den quergestreiften Muskeln. Die Befunde, die unter Ausnutzung der Enzymnatur des Myosins mit einer histochemischen Methode im elektronenmikroskopischen Maßstab erhoben wurden, teilen wir im folgenden in kurzer Form mit.

Zum Nachweis der ATPase-Aktivität des Myosins bedienten wir uns im wesentlichen der bereits früher angewendeten Technik ${ }^{14,15}$. Kleine Stücke des Hautmuskelschlauchs wurden unmittelbar nach der Präparation oder nach 12-24-stdg. Extraktion mit Glycerin für wenige Min. in eiskaltes Fixierungsgemisch gebracht und nach kurzem Waschen mit $0,154-m$. KCl 10 bis $30 \mathrm{Min}$. bei $20^{\circ} \mathrm{C}$ inkubiert. In manchen Versuchen kamen auch Gefrierschnitte zur Verwendung. Als Inkubationsmedien dienten modifizierte Gemische nach Mölbert, Duspiva und v. Deimling ${ }^{17}$ oder nach Wachstein und Meisel ${ }^{18}$. Die Proben wurden darauf 90 Min. mit $\mathrm{OsO}_{4}{ }^{19}$ fixiert, entwässert und in Araldit eingebettet. Die Ultradünnschnitte wurden mit einem LKB Ultrotome hergestellt, mit Uranylacetat kontrastiert ${ }^{20}$ und mit einem Zeiss EM 9 untersucht.

Die elektronenmikroskopische Prüfung der zum ATPase-Nachweis inkubierten Präparate ergab eine

16 H.-G. Heumann u. E. Zebe, unveröffentlicht.

17 E. R. G. Mölbert, F. Duspiva u. O. H. v. Deimling, J. biophysic. biochem. Cytol. 7, 387 [1960].

18 M. W [1957]. charakteristische Verteilung des durch die Enzymwirkung freigesetzten und mit Blei gefällten anorganischen Phosphats. Dies demonstrieren Querschnitte besonders eindrucksvoll (Abb. $1^{*}$ ). Es finden sich hier sehr regelmäßige Muster aus Zonen, die mit Präzipitat dicht bedeckt, und solchen, die praktisch frei davon sind. Ein Vergleich der Querschnitte mit den verschiedenen Arten von Längsschnitten (sagittal und frontal) läßt erkennen, daß die Bleiphosphatpartikel stets auf den Bereich der dicken Filamente beschränkt sind. Das wird auf Sagittalschnitten besonders deutlich, weil die Anordnung der Filamente dann der Organisation der Myofibrillen aus quergestreiften Muskeln gleicht (Abb. 2). Die dicken Filamente erscheinen hier in Verbänden, die den A-Banden jener entsprechen, wenngleich sie weniger exakt ausgerichtet sind.

Die Kristallite sind in der zentralen Zone eines jeden „A-Bandes“ am stärksten massiert. Sie liegen hier reihenweise in den Zwischenräumen zwischen den einzelnen dicken Filamenten und füllen diese nicht selten (insbesondere bei Anwendung des Mediums nach $\mathrm{W}$ achstein und $\mathrm{Meisel)}$ völlig aus. Die Randzonen, die an beiden Seiten etwa 20 bis $30 \%$ der Gesamtlänge der dicken Filamente einnehmen, enthalten dagegen deutlich weniger Niederschlag. In Präparaten mit geringer Spaltungsintensität finden sich hier nur vereinzelte Kristallite. Die den I-Banden der quergestreiften Muskeln entsprechenden Bereiche zwischen den Verbänden der dikken Filamente, in denen sich ausschließlich dünne Filamente finden, sind praktisch frei von Präzipitat. Frontalschnitte zeigen, daß die auf den Sagittalschnitten beobachteten Verbände von dicken Filamenten, die „A-Banden“, jeweils aus nur einer Schicht bestehen und daß jede Schicht gegen die nächste stark versetzt ist. Dadurch liegt ein Ende jedes dicken Filaments auf der einen Seite des aus mehreren (meist 3 oder 4) Schichten gebildeten Filamentbündels, das andere auf der gegenüberliegenden. Die Filamentbündel zeigen infolgedessen einen schrägen Verlauf, während die einzelnen Filamente parallel zur Längsachse der Faser orientiert sind.

19 G. E. Palade, J. exp. Medicine 95, 285 [1952].

20 M. W

* Abbn. 1-4 s. Tafel S. 64 a. 
Der Bleiphosphatniederschlag findet sich überwiegend auf das Innere der Filamentbündel beschränkt, liegt also in erster Linie im Zwischenraum zwischen zwei dicken Filamenten (Abb. 3). Die dem Zwischenraum zwischen den Bündeln zugekehrte Seite der dicken Filamente, an die nur dünne Filamente grenzen, ist häufig fast frei von Niederschlag oder enthält wenigstens sehr viel weniger davon als die einem anderen dicken Filament zugekehrte Seite. Diese Verteilung entspricht dem im Sagittalschnitt beobachteten Bild des „ABandes" mit einer zentralen Zone von großer Niederschlagsdichte und Randzonen mit deutlich vermindertem Präzipitat. Gelegentlich ist auf Frontalschnitten auch eine auffallende Regelmäßigkeit in der Anordnung der einzelnen Bleiphosphatpartikel zu erkennen. Diese liegen dann nicht nur in longitudinal orientierten Reihen, sondern haben innerhalb der Reihen auch annähernd gleichmäßige Abstände von schätzungsweise $350 \AA$.

Die bisher geschilderten Befunde beziehen sich auf Muskeln, die im erschlafften Zustand vorfixiert wurden. Bei der Kontraktion werden die dünnen Filamente offenbar in der gleichen Weise zwischen die dicken gezogen wie bei den quergestreiften Muskeln, denn der Abstand zwischen den Bündeln der dicken Filamente verringert sich. Im Gegensatz zu jenen verschieben sich aber auch die einzelnen Schichten der dicken Filamente gegeneinander, und zwar in dem Sinne, daß sich der gegenseitige Versetzungsgrad verringert. Dies führt zu einer Vermehrung der Zahl von Filamentschichten, die ein Bündel bilden.

Die Demonstration der ATPase-Aktivität in solchen Muskeln ist äußerst schwierig, weil die Diffusion von Substrat und Fällungsreagenz offenbar stark erschwert ist. Gelingt sie jedoch, dann zeigen die Frontalschnitte entsprechend der Verbreiterung der Filamentbündel auch eine erheblich verbreiterte zentrale Reaktionszone (Abb.4). Die auslaufenden Enden der dicken Filamente, die bei starker Kontraktion leicht S-förmig abgebogen sind, enthalten dagegen praktisch keinen Niederschlag.

Bei allen Experimenten wurden Kontrollen ohne Substrat angesetzt. Darin fand sich gelegentlich ebenfalls eine geringfügige Präzipitation. Sie ist vermutlich auf die Spaltung von zelleigenem ATP zurückzuführen, denn Glycerin-extrahierte Präparate oder Gefrierschnitte, bei denen die löslichen Komponenten weitgehend ausgewaschen werden, lassen niemals Spuren davon erkennen (vgl. l. c. ${ }^{14,15}$ ). Mit Reagenzien, die SH-Gruppen blockieren, wie $N$-Äthylmaleinimid $(0,01-m$. $)$ läßt sich eine vollständige Hemmung der ATP-Spaltung erreichen. Die beobachtete Präzipitation von Bleiphosphat kann demnach mit großer Wahrscheinlichkeit auf die enzymatische Spaltung von ATP zurückgeführt werden.

Aus den geschilderten Befunden geht eindeutig hervor: 1. Die Präzipitation von Bleiphosphat im kontraktilen Apparat der Fasern aus dem Hautmuskelschlauch des Regenwurms, die zum ATPaseNachweis inkubiert wurden, findet ausschließlich in unmittelbarer Nähe der dicken Filamente statt. 2. Diese Verteilung gilt für alle Kontraktionsstadien. Die dicken Filamente müssen demnach Sitz des ATP. spaltenden Enzyms sein.

In den quergestreiften Muskeln sind die dicken Filamente mindestens überwiegend aus Myosin aufgebaut ${ }^{10-15}$. Da das aus Muskeln des Regenwurms isolierte Myosin dem aus Vertebratenmuskeln offenbar weitgehend ähnlich ist ${ }^{21}$ und da darüber hinaus der Kontraktionsvorgang, in dessen Verlauf ja das ATP gespalten wird, bei beiden anscheinend essentiell in der gleichen Weise abläuft, darf gefolgert werden, daß 1. Myosin mit großer Wahrscheinlichkeit auch für die ATP-Spaltung im Regenwurm-

21 K. Maruyama u. D. R. Kominz, Z. vgl. Physiol. 42, 17 [1959].

Abb. 1. Längsmuskulatur des Hautmuskelschlauchs von Lumbricus im Querschnitt. Vorfixierung mit Formol (5-proz.)/ Veronal $0,3-m$., Inkubation der Gefrierschnitte für $20 \mathrm{~min}$ nach l.c. ${ }^{17}$. Verteilung des Bleiphosphatniederschlags in regelmäßigen Bändern, die die Position der Bündel von dicken Filamenten anzeigen.

Abb. 2. Sagittalschnitt einer mit Glycerin extrahierten Faser. Vorfixierung mit Calcium/Formol, 10 min Inkubation nach l. c. ${ }^{17}$. Auffällige Massierung der Kristallite im mittleren Bereich der „A-Banden“ (A).
Abb. 3. Frontalschnitt eines erschlafften Muskels. Vorfixierung mit ungepuffertem, neutralisiertem Formol, 30 min Inkubation nach 1. c. ${ }^{18}$. Der Niederschlag ist auf die unmittelbare Nähe der dicken Filamente beschränkt.

Abb. 4. Frontalschnitt eines kontrahierten Muskels. Vorbehandlung wie in Abb. 1. Die Bündel der dicken Filamente und die Reaktionszonen sind im Vergleich zum erschlafften Muskel verbreitert.

Der Maßstab entspricht jeweils $1 \mu \mathrm{m}$. 


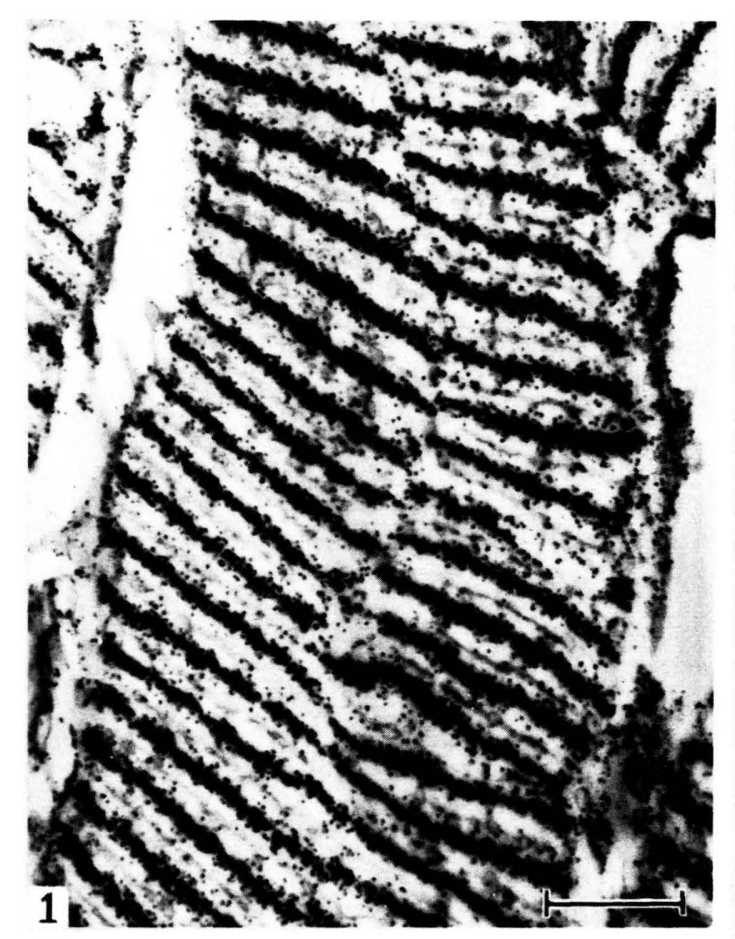

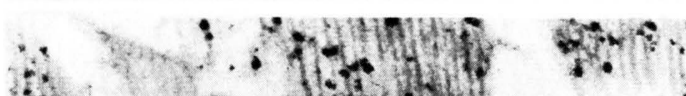

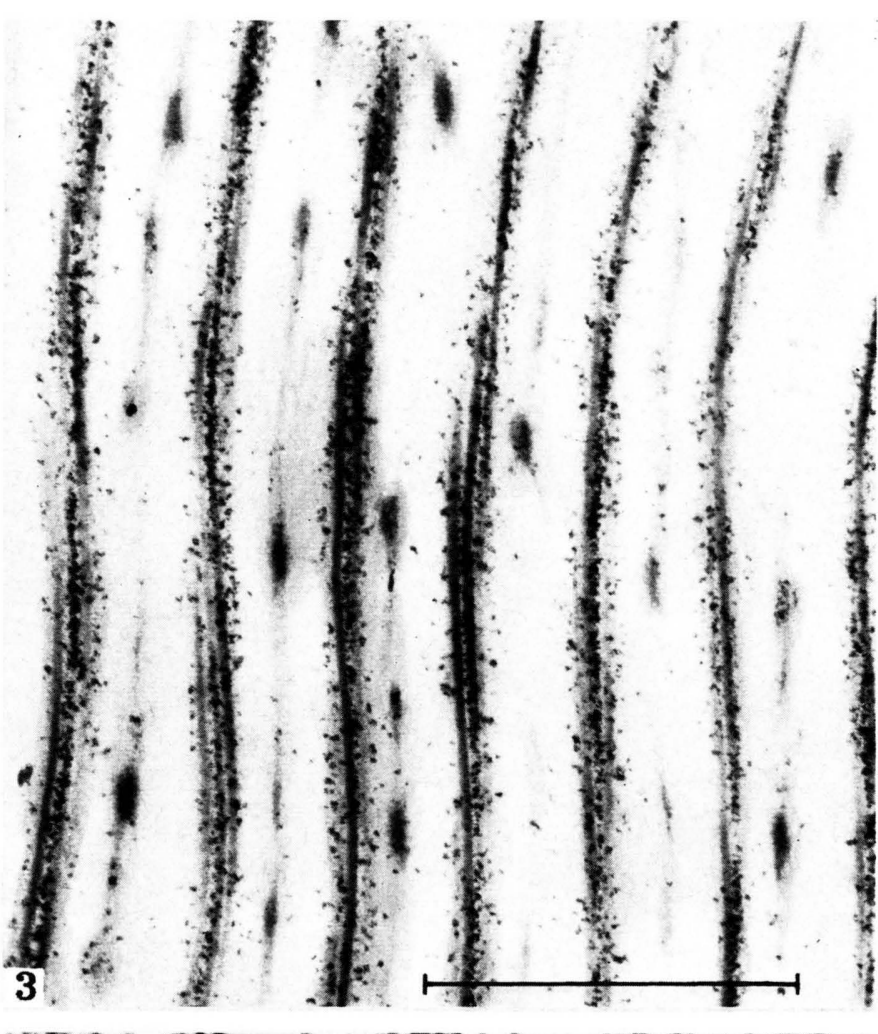

E.

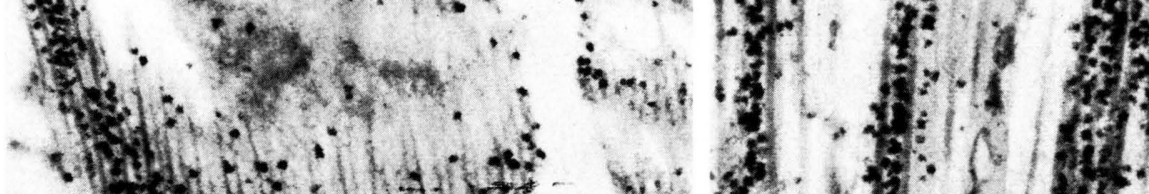

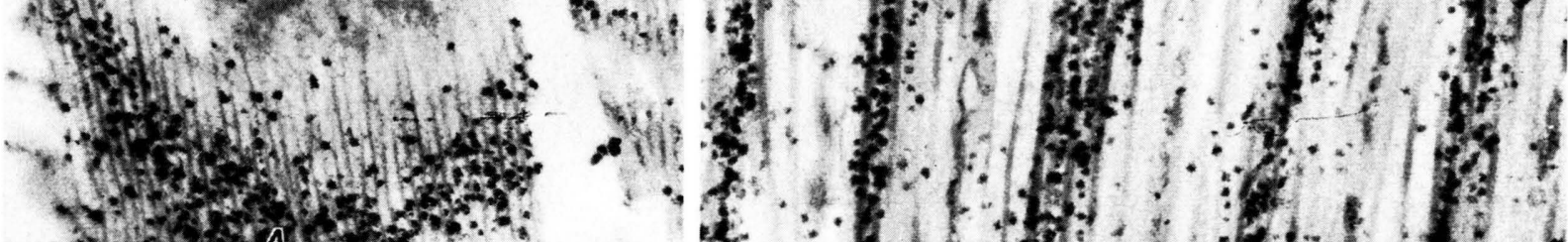

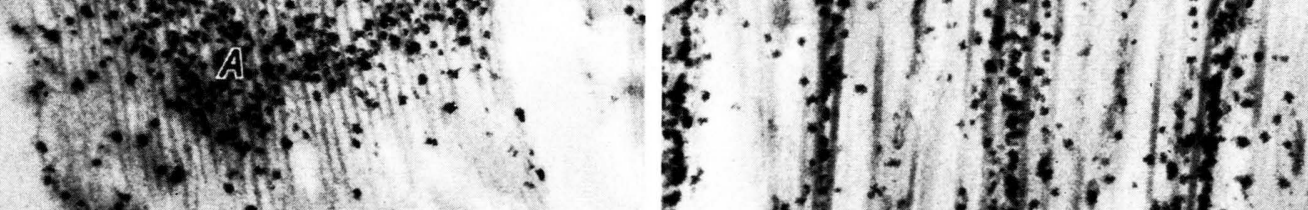

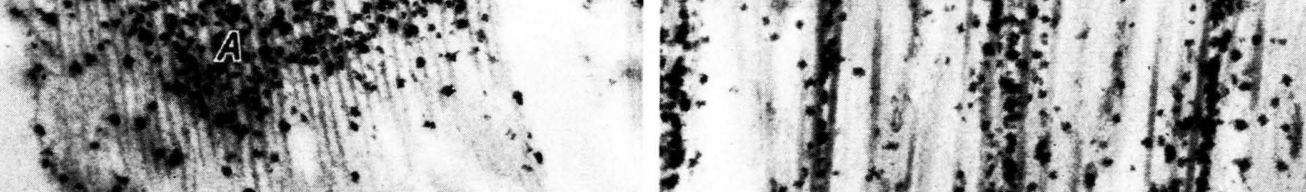

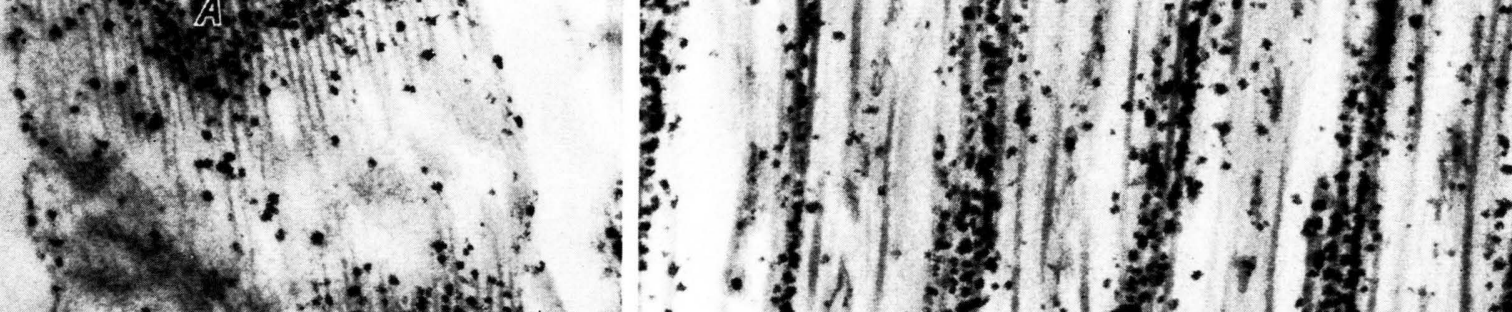
If

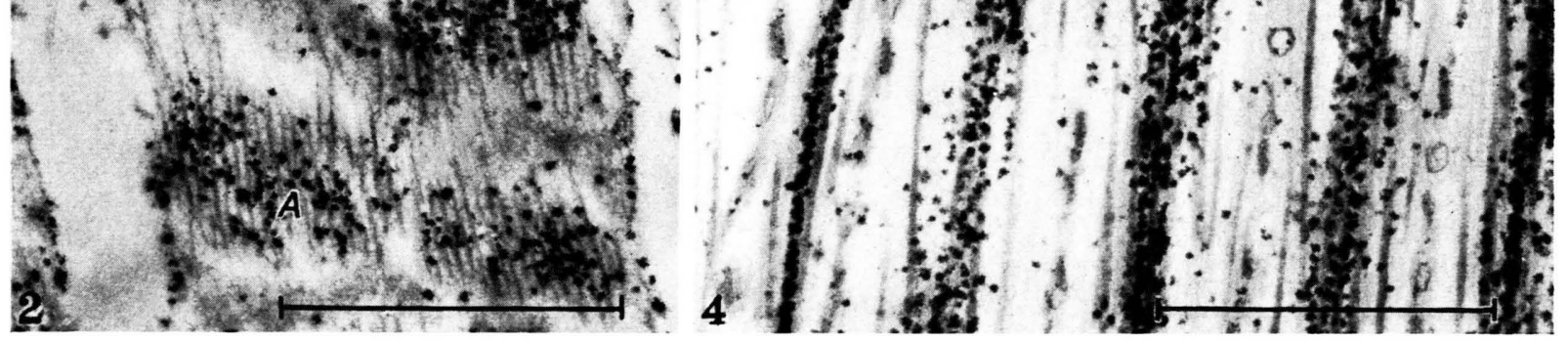



muskel verantwortlich ist und daß 2. dieses kontraktile Protein gleichfalls in den dicken Filamenten lokalisiert ist.

Übereinstimmung mit den quergestreiften Muskeln herrscht weiterhin im Hinblick auf das Vorliegen maximaler Spaltungsaktivität im Mittelteil der dicken Filamente, von dem sich weniger aktive Randzonen abheben ${ }^{15}$. Ob die Ursache hierfür im Aufbau der Filamente oder aber in der im Bereich der Randzonen erschwerten Knüpfung von Querbrücken zu den Aktinfilamenten zu suchen ist, läßt sich vorerst nicht entscheiden. Eine der H-Zone der quergestreiften Muskeln vergleichbare Region, in der nur Myosinfilamente vorliegen und die Aktinfilamente fehlen, läßt sich auf Querschnitten zwar im Zentrum der Bündel von dicken Filamenten erkennen. Der Nachweis, daß darin ebenso wie in den H-Zonen jener keinerlei Spaltung von ATP stattfindet ${ }^{15}$, ist allerdings bisher noch nicht gelungen.

Der gelegentlich beobachteten longitudinalen Periodizität in der Ablagerung der Bleiphosphatpartikel an den dicken Filamenten liegt möglicherweise eine longitudinale Periodizität der ATP-Spaltung zugrunde. Nach den Vorstellungen HuxLeys wird ATP bei der Knüpfung bzw. Lösung der Querbrücken zwischen den Myosin- und Aktinfilamenten gespalten ${ }^{1}$. Solche Querbrücken sind zwar für Regenwurmmuskeln bislang noch nicht nachgewiesen worden, doch ist anzunehmen, daß sie auch hier existieren. Die periodische Anordnung der Bleiphosphatkristallite ist vielleicht ein Anzeichen für ihre Existenz, denn in ähnlichen Experimenten an Fibrillen aus Herzmuskel konnte eine 400 Å-Periodizität der Niederschlagsablagerung beobachtet werden, die

22 L. W. Tice u. R. J. Barrnett, J. Cell Biol. 15, 401 [1962].

23 I. Pucci u. B. A. Afzelius, J. Ultrastructure Res. 7, 210 [1962]. dem für diese Muskeln gemessenen Abstand zwischen den einzelnen Querbrücken entspricht ${ }^{22,1}$.

Schließlich muß noch auf Strukturen eingegangen werden, die jeweils in der Mitte zwischen den Bündeln der dicken Filamente liegen. Es sind dies 1. solide Gebilde mit verschwommenem Umriß und 2. englumige Vesikel oder Tubuli mit scharf abgesetzten Membranen. Beide durchziehen die Muskelfasern in transversaler Richtung und wechseln mehr oder weniger regelmäßig miteinander ab. Die Tubuli sind höchstwahrscheinlich Teile des sarcoplasmatischen Reticulums. Unter bestimmten Bedingungen findet sich auch in ihnen präzipitiertes Bleiphosphat ${ }^{16}$. Natur und Funktion der soliden Strukturen sind dagegen umstritten. Pucci und Afzelius nehmen an, sie seien „compact sarcotubules“, d. h. gleichfalls Komponenten des sarcoplasmatischen Reticulums ${ }^{23}$. Nach RöHLIch stellen sie vielleicht kontraktile Elemente („cross-filaments“) dar, die sich gleichzeitig mit den longitudinalen kontrahieren und damit einer zu starken Verdickung der Fasern entgegenwirken ${ }^{24}$. Ікемото sieht in ihnen schließlich Bildungen, die den Z-Scheiben der quergestreiften Muskeln entsprechen und an denen die dünnen Filamente ansetzen ${ }^{6}$. Da wir niemals Spuren von Bleiphosphat in der Nähe dieser Strukturen beobachtet haben, halten wir die Deutung Iкемотоs für die wahrscheinlichste.

Zusammenfassend kann festgestellt werden, daß die Fasern aus dem Hautmuskelschlauch des Regenwurms den quergestreiften Muskeln im Hinblick auf Feinbau und Funktionsweise sehr viel näher stehen, als dies zunächst erscheinen mag.

24 P. Röhlich, J. Ultrastructure Res. 7, 399 [1962]. 